\title{
Calcific uraemic arteriolopathy (calciphylaxis) in patients on renal replacement therapy
}

\author{
S Sebastian, ${ }^{1}$ MB ChB, FCP (SA), MMed (Internal Medicine), Cert Nephrology (SA) Phys, MPhil (Nephrology); \\ H F Jordaan, ${ }^{2} \mathrm{MB}$ ChB, MMed (Dermatology); J W Schneider, ${ }^{3} \mathrm{MB}$ ChB, MMed (Anatomical Pathology), FCPath; \\ M R Moosa, ${ }^{1}$ MB ChB, FCP (SA), MD, FRCP (Lond); M R Davids, ${ }^{1}$ MB ChB, FCP (SA), MMed (Internal Medicine), PhD

\begin{abstract}
${ }^{1}$ Division of Nephrology, Department of Medicine, Faculty of Medicine and Health Sciences, Stellenbosch University and Tygerberg Hospital, Cape Town, South Africa

${ }^{2}$ Division of Dermatology, Faculty of Medicine and Health Sciences, Stellenbosch University and Tygerberg Hospital, Cape Town, South Africa ${ }^{3}$ Division of Anatomical Pathology, National Health Laboratory Service, Faculty of Medicine and Health Sciences, Stellenbosch University and Tygerberg Hospital, Cape Town, South Africa
\end{abstract}

Corresponding author: M R Davids (mrd@sun.ac.za)

Background. Calcific uraemic arteriolopathy (calciphylaxis) is an unusual and potentially fatal condition characterised by small-vessel calcification and ischaemic skin necrosis. It mainly affects patients with end-stage renal disease (ESRD) on haemodialysis, but may rarely occur in the absence of ESRD in conditions such as primary hyperparathyroidism, malignancy, alcoholic liver disease and connective tissue disease. Methods. We reviewed the records of all patients diagnosed with calciphylaxis while on renal replacement therapy at Tygerberg Hospital, Cape Town, South Africa, between 1990 and 2014, to describe its presentation, course and final outcome.

Results. Nineteen patients developed calciphylaxis over this period. Their median age was 34 years and 13 (68.4\%) were female. Fifteen (78.9\%) had received a kidney transplant. All patients had painful skin lesions that rapidly progressed to infarction. Small-vessel calcification was seen on skin biopsy in 13 patients. Twelve patients had hyperparathyroidism. Several of the transplanted patients had been treated for graft rejection in the year preceding the diagnosis. Treatment consisted of good wound care and efforts to normalise serum calcium and phosphate levels. Five patients received an urgent parathyroidectomy. The outcome was fatal in 17 patients, with sepsis being the main cause of death.

Conclusions. In our patients, calciphylaxis carried a worse prognosis than previously reported internationally. It should always be considered in the differential diagnosis of painful skin lesions in the dialysis or transplant patient.

S Afr Med J 2017;107(2):140-144. DOI:10.7196/SAMJ.2017.v107i2.11058

Calcific uraemic arteriolopathy (systemic calciphylaxis) is a rare, lifethreatening condition characterised pathologically by small-vessel calcification and ischaemic skin necrosis. ${ }^{[1]}$ It occurs most frequently in the setting of end-stage renal diasease (ESRD) with secondary hyperparathyroidism and is more common in females than in males. In patients without advanced renal disease, it has been reported in cases of primary hyperparathyroidism, malignancy, alcoholic liver disease and connective tissue disease. ${ }^{[2]}$

The skin lesions of calciphylaxis typically develop suddenly and progress rapidly. Lesions may be singular or numerous, and generally occur on the legs. Intense pain is almost invariable. Early lesions of calciphylaxis manifest with dusky erythema, violaceous mottling or livedo reticularis, and progress to indurated papules, plaques or nodules. More developed skin lesions have an irregular outline that gives a stellate, purpuric appearance with central necrosis (see Fig. 1). Secondary infection of ulcerated skin lesions and subsequent fulminant sepsis are responsible for the significant morbidity and mortality associated with this condition.

Most cases have been described in patients with ESRD who are treated with renal replacement therapy (RRT) in the form of chronic dialysis or kidney transplantation. A cross-sectional study in patients on chronic haemodialysis reported a $4 \%$ prevalence of calciphylaxis. ${ }^{[3]}$ Calciphylaxis complicating non-uraemic disorders is much rarer, with a systematic review published in $2008^{[2]}$ reporting a total of only 36 cases. Of these patients, 10 had kidney disease as indicated by elevated serum creatinine concentrations; in 12 cases the serum creatinine level was not reported.

The pathogenesis of the condition remains uncertain. The term calciphylaxis was first coined by Hans Selye, who described an experimental rat model of metastatic calcification. His model seldom produced vascular calcification, however, and did not produce the skin necrosis so typical of the disease in humans. ${ }^{[4]}$ Cutaneous vascular stenosis and thrombotic occlusion are separate processes that are both required to produce the lesions of calciphylaxis. ${ }^{[5]}$ Vascular stenosis is caused by medial arteriolar calcification and subintimal fibrosis. Risk factors such as chronic inflammation, hyperparathyroidism, and drugs such as glucocorticoids and calcineurin inhibitors are frequently present in patients with ESRD and may lead to activation of nuclear factor $\kappa-B(N F \kappa B) .{ }^{[5]}$ This promotes the transdifferentiation of vascular smooth-muscle cells to an osteogenic phenotype with subsequent vascular calcification. The vascular thrombosis occurs abruptly, precipitating the presentation of patients with the typical painful ischaemic skin lesions. Low flow rates through narrowed vessels, damage by subintimal fibrosis, and hypercoagulability from local and/or systemic inflammation and endothelial dysfunction may all contribute to vascular thrombosis. ${ }^{[5]}$

The diagnosis of calciphylaxis in patients being treated with RRT for ESRD is based mainly on the clinical presentation with the characteristic skin lesions. ${ }^{[6,7]}$ A skin biopsy can worsen the skin lesions and need not be performed routinely. Obtaining a biopsy 
specimen is recommended if the clinical features are not typical and when alternative diagnoses, such as warfarin skin necrosis or diabetic gangrene, are being considered. ${ }^{[6,7]}$ Histopathological findings include medial calcification of small vessels, intimal proliferation, thrombosis, soft-tissue calcification, panniculitis and skin necrosis. Demonstrating calcifications in other vascular beds on plain radiographs is probably not useful in making the diagnosis of calciphylaxis, as this is a common finding in many patients on RRT.

Risk factors for developing calciphylaxis include female gender, white ethnicity, diabetes mellitus, obesity and secondary hyperparathyroidism. ${ }^{[8-11]}$ Triggers such as the use of vitamin D preparations, calcium-based phosphate binders, corticosteroids, parenteral iron supplementation and warfarin have been postulated to initiate calcification in the 'at-risk' individual. ${ }^{[9,12]}$

The optimal treatment of the condition remains uncertain. ${ }^{[13]}$ Principles of therapy include optimal wound care (sterile dressings, debridement, treating secondary infection), nutritional support in malnourished patients, and attempts to normalise serum calcium and phosphate levels to lower the calcium-phosphate product. Parathyroidectomy has resulted in dramatic improvement in some cases, but the response is inconsistent and unpredictable. ${ }^{[14]}$ Other treatment strategies include the use of non-calcium-based phosphate binders, bisphosphonates ${ }^{[13,15]}$ and cinacalcet. ${ }^{[16]}$ Intensified haemodialysis, hyperbaric oxygen ${ }^{[17]}$ and sodium thiosulphate, the antioxidant cation chelator ${ }^{[18]}$ used as a component of multimodal therapy, may have contributed to the favourable outcome in some series. ${ }^{[19]}$

In this article we describe 19 patients with ESRD who presented with calciphylaxis, the majority of whom had received a renal transplant. Patients were managed with multimodal therapy as available in our resource-constrained setting. This involved cessation of calcium-based phosphate binders and vitamin $\mathrm{D}$, increased efforts to address the hyperphosphataemia and optimise haemodialysis, good wound care and antibiotics. A parathyroidectomy was performed in selected cases and a single patient received bisphosphonate treatment.

\section{Methods}

The medical records of patients receiving RRT who were diagnosed with calciphylaxis in the Division of Nephrology at Tygerberg Hospital, Cape Town, South Africa, between 1990 and 2014 were reviewed. The records of all patients admitted to our RRT programme as well as those who have died are housed in the division. Only the clinicians who were directly involved in the routine clinical care of the patients described in this study accessed these records. Patient demographics, the clinical presentation, potential risk factors and laboratory results, including skin biopsy data, were extracted and recorded on standardised data capture sheets.

A clinical diagnosis of calciphylaxis was made in patients with ESRD who presented with the typical non-healing ischaemic skin lesions and who had no obvious alternative diagnosis. Diagnostic criteria have been proposed by Hiyashi ${ }^{[6]}$ and require the following clinical criteria: a patient with ESRD; more than two painful and nontreatable skin ulcers with concomitant painful purpura; and lesions on the trunk, extremities, or penis with concomitant painful purpura. A diagnosis can also be made when two of these three clinical features are present with typical histopathological findings.

\section{Ethics approval}

As this was a retrospective study, a waiver of individual informed consent was granted by the Health Research Ethics Committee at Stellenbosch University.

\section{Results}

Tygerberg Hospital is a 1384 -bed public sector teaching hospital. The number of patients on RRT is limited to 70 patients on haemodialysis and 60 patients on peritoneal dialysis owing to resource constraints. In addition, we follow up $\sim 160$ kidney transplant patients. Only patients who are suitable for kidney transplantation are accommodated, and peritoneal dialysis is usually the first treatment modality. Few diabetic patients are accommodated because of the frequent presence of multiple diabetic complications on presentation and poorer outcome following kidney transplantation. During the period 1990 - 2014, 937 new patients $(53.0 \%$ female) were admitted to the renal replacement programme. Most of the patients $(n=626,66.8 \%)$ were of mixed ethnicity, while 151 (16.1\%) were white, 141 (15.9\%) black and 19 (2.0\%) Indian.

Nineteen patients ( $2.0 \%$ of those admitted to the RRT programme) were diagnosed with calciphylaxis (Table 1). This group had a median age of 34 years (interquartile range (IQR) 29 - 40) and consisted of 13 females and 6 males. Most patients were white $(n=12,63.2 \%), 6$ were of mixed ancestry and only 1 was black. Three were obese (all female), but none was diabetic. Chronic glomerulonephritis was the most commonly reported cause of ESRD (11 patients).

All patients presented with typical painful, purpuric skin lesions. Ulceration was either present at presentation or developed later in the course of the disease. The lesions were typically indurated plaques and nodules (11 patients), were sometimes associated with livedo reticularis, and were confined to the legs below the knees in $73.7 \%$ of cases (Fig. 1). Two patients presented with proximal lesions (thighs, buttocks, abdomen) and 3 with both proximal and distal lesions. Most patients had radiographic evidence of vascular calcification (Fig. 2). Skin biopsy data were available in 18 patients, and in 13 the biopsy findings supported the diagnosis of calciphylaxis, revealing small-vessel calcification, panniculitis and, in one case, microvascular thrombosis (Figs 3 and 4).

The median time to diagnosis of calciphylaxis from the initiation of dialysis for the group was 51 months (IQR 12 - 96). Fifteen patients had previously received a renal transplant, with a median time to transplant of 29 months (IQR 4 - 60) after starting dialysis. In these patients, calciphylaxis was diagnosed at a median of 26 months (IQR 11 - 79) after transplantation. Six patients still had a functioning graft at the time of diagnosis.

Twelve patients (63.2\%) had laboratory evidence of hyperparathyroidism (defined by serum intact parathyroid hormone concentrations $>8.4 \mathrm{pmol} / \mathrm{L}$, the upper limit of normal for our laboratory). The calcium-phosphate product was $4.4 \mathrm{mmol}^{2} / \mathrm{L}^{2}$ in 10 of these patients. All patients were receiving our standard regimen of calciumbased phosphate binders and vitamin $\mathrm{D}$ for the treatment of secondary hyperparathyroidism. The 6 patients with functioning kidney transplants were on our standard immunosuppression regimen of prednisone, azathioprine and cyclosporine. Five of the 15 previously transplanted patients had been pulsed with intravenous steroids (methylprednisolone) for graft rejection in the year preceding the diagnosis of calciphylaxis. Two patients had used warfarin in the past (Table 1, patients 5 and 7), 1 (patient 8) was on long-term warfarin treatment for previous pulmonary embolism at the time of the diagnosis of calciphylaxis, and 7 had received intravenous iron supplementation during the months leading up to their presentation with calciphylaxis.

Multimodal therapy for all patients included cessation of calciumbased phosphate binders and vitamin $\mathrm{D}$, strict dietary phosphate restriction, intensified haemodialysis (including more frequent 
Table 1. Clinical features of 19 patients with calciphylaxis

\begin{tabular}{|c|c|c|c|c|c|c|c|c|c|c|c|c|}
\hline $\begin{array}{l}\text { Patient } \\
\text { no. }\end{array}$ & Sex & Age & $\begin{array}{l}\text { Ethnic } \\
\text { group }\end{array}$ & Renal dx & Modality & Site & $\begin{array}{l}\text { PTH } \\
(\mathrm{mmol} / \mathrm{L})\end{array}$ & $\begin{array}{l}\mathrm{Ca} \\
(\mathrm{mmol} / \mathrm{L})\end{array}$ & $\begin{array}{l}\mathrm{PO}_{4} \\
(\mathrm{mmol} / \mathrm{L})\end{array}$ & XR & Bx & Outcome \\
\hline 1 & $\mathrm{~F}$ & 29 & Mixed & $\begin{array}{l}\text { Chronic } \\
\text { GN }\end{array}$ & Tx-HD & Dist. & 170 & 2.38 & 1.84 & Y & $\mathrm{Y}$ & Died (sepsis) \\
\hline 2 & $\mathrm{~F}$ & 25 & White & SLE & HD-Tx & Prox. & 39.6 & 2.64 & 3.42 & $\mathrm{Y}$ & $\mathrm{N}$ & $\begin{array}{l}\text { Died (CNS } \\
\text { bleed) }\end{array}$ \\
\hline 3 & M & 49 & Mixed & MHT & $\begin{array}{l}\text { PD-Tx- } \\
\text { HD }\end{array}$ & Dist. & NA & 2.44 & 2.11 & NA & Y & Died (GI bleed) \\
\hline 4 & $\mathrm{~F}$ & 31 & White & Reflux & $\begin{array}{l}\text { Tx-PD- } \\
\text { Tx }\end{array}$ & Prox. & 98 & 2.59 & 2.48 & Y & $\mathrm{Y}$ & Died (sepsis) \\
\hline 5 & $\mathrm{~F}$ & 38 & Black & $\begin{array}{l}\text { Chronic } \\
\text { GN }\end{array}$ & Tx-PD & Dist. & 43.7 & 2.94 & 3.36 & $\mathrm{Y}$ & $\mathrm{N}$ & Died (sepsis) \\
\hline 6 & $\mathrm{~F}$ & 40 & White & $\begin{array}{l}\text { Chronic } \\
\text { GN }\end{array}$ & PD-HD & Dist. & 123 & 2.7 & 3.45 & $\mathrm{~N}$ & Y & Died (sepsis) \\
\hline 7 & M & 39 & Mixed & $\begin{array}{l}\text { Chronic } \\
\text { GN }\end{array}$ & HD-Tx & Dist. & 8 & 2.68 & NA & $\mathrm{N}$ & Y & Sudden death \\
\hline 8 & $\mathrm{~F}$ & 42 & White & Reflux & $\begin{array}{l}\text { PD-Tx- } \\
\text { HD }\end{array}$ & Dist. & NA & 2.18 & 2.55 & $\mathrm{Y}$ & NA & Died (sepsis) \\
\hline 9 & $\mathrm{~F}$ & 40 & White & $\begin{array}{l}\text { Chronic } \\
\text { GN }\end{array}$ & $\begin{array}{l}\text { HD-PD- } \\
\text { HD-Tx }\end{array}$ & Dist. & 16 & 2.53 & 0.86 & Y & $\mathrm{N}$ & Died (sepsis) \\
\hline 10 & $\mathrm{~F}$ & 34 & White & ADPKD & HD & Dist. & 44 & 1.99 & 5.31 & Y & $\mathrm{N}$ & Died (MI) \\
\hline 11 & $\mathrm{~F}$ & 34 & Mixed & $\begin{array}{l}\text { Chronic } \\
\text { GN }\end{array}$ & PD-Tx & Dist. & 16.4 & 2.47 & 1.5 & Y & Y & Died (sepsis) \\
\hline 12 & $\mathrm{~F}$ & 29 & White & $\begin{array}{l}\text { Chronic } \\
\text { GN }\end{array}$ & ESRD $^{*}$ & $\begin{array}{l}\text { Prox./ } \\
\text { dist. }\end{array}$ & $201+$ & 1.79 & 2.56 & Y & $\mathrm{Y}$ & Alive \\
\hline 13 & M & 32 & White & $\begin{array}{l}\text { Chronic } \\
\text { GN }\end{array}$ & $\begin{array}{l}\text { PD-Tx- } \\
\text { HD }\end{array}$ & Dist. & $201+$ & 2.11 & 3.21 & Y & $\mathrm{N}$ & Alive \\
\hline 14 & $\mathrm{~F}$ & 34 & Mixed & $\begin{array}{l}\text { Chronic } \\
\text { GN }\end{array}$ & $\mathrm{PD}$ & $\begin{array}{l}\text { Prox./ } \\
\text { dist. }\end{array}$ & 102 & 2.68 & 2.4 & Y & Y & Died (sepsis) \\
\hline 15 & M & 38 & White & IN & $\begin{array}{l}\text { PD-Tx- } \\
\text { HD-Tx- } \\
\text { PD }\end{array}$ & Dist. & $201+$ & 2.08 & 3.06 & Y & Y & Died (sepsis) \\
\hline 16 & $\mathrm{~F}$ & 29 & White & $\begin{array}{l}\text { Chronic } \\
\text { GN }\end{array}$ & $\begin{array}{l}\text { PD-Tx- } \\
\text { PD-Tx- } \\
\text { PD }\end{array}$ & $\begin{array}{l}\text { Prox./ } \\
\text { dist. }\end{array}$ & NA & 2.14 & 2.45 & Y & Y & Died (sepsis) \\
\hline 17 & M & 58 & Mixed & ADPKD & PD-Tx & Dist. & NA & 1.74 & 3.06 & Y & $\mathrm{Y}$ & Died (sepsis) \\
\hline 18 & M & 45 & White & Reflux & Tx-PD & Dist. & NA & NA & NA & NA & Y & Died (sepsis) \\
\hline 19 & $\mathrm{~F}$ & 14 & White & $\begin{array}{l}\text { Chronic } \\
\text { GN }\end{array}$ & Tx-PD & Dist. & NA & 2.52 & 2.02 & $\mathrm{~N}$ & Y & Died (sepsis) \\
\hline
\end{tabular}

haemodialysis), use of low-calcium dialysate, aggressive wound care (daily dressings, debridement) and antibiotic cover. Five patients had an urgent parathyroidectomy (patients 1, 12, 13, 14 and 16). One patient also received a course of intravenous bisphosphonates, and demonstrated good wound healing over a period of 2 months.

Four patients were considered to have been cured with aggressive multimodal therapy. Two of them (patients 7 and 10) subsequently died suddenly of cardiovascular causes, one within a month of disease stabilisation and the other after 22 months. The overall survival at 6 months after the diagnosis of calciphylaxis was $31.6 \%$, and at 1 year the survival rate was $15.8 \%$. The median time from diagnosis to death for the 15 patients who died from complications of calciphylaxis was 9 weeks (IQR 3 - 17). Most of the deaths were the result of severe sepsis related to infection of the necrotic skin lesions.

\section{Discussion}

Our study confirms that calciphylaxis is a relatively rare disease, affecting $\sim 2 \%$ of our patients on RRT, and that it has a very poor prognosis. A particularly striking finding was that our white patients were much more frequently affected (7.9\%) than our black patients 


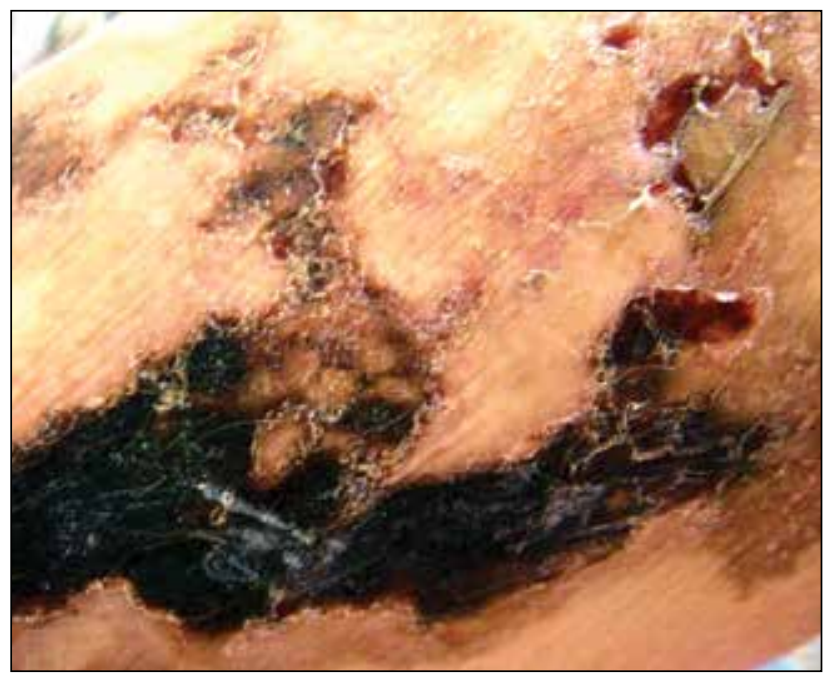

Fig. 1. Ischaemic skin necrosis with the typical irregular outline.

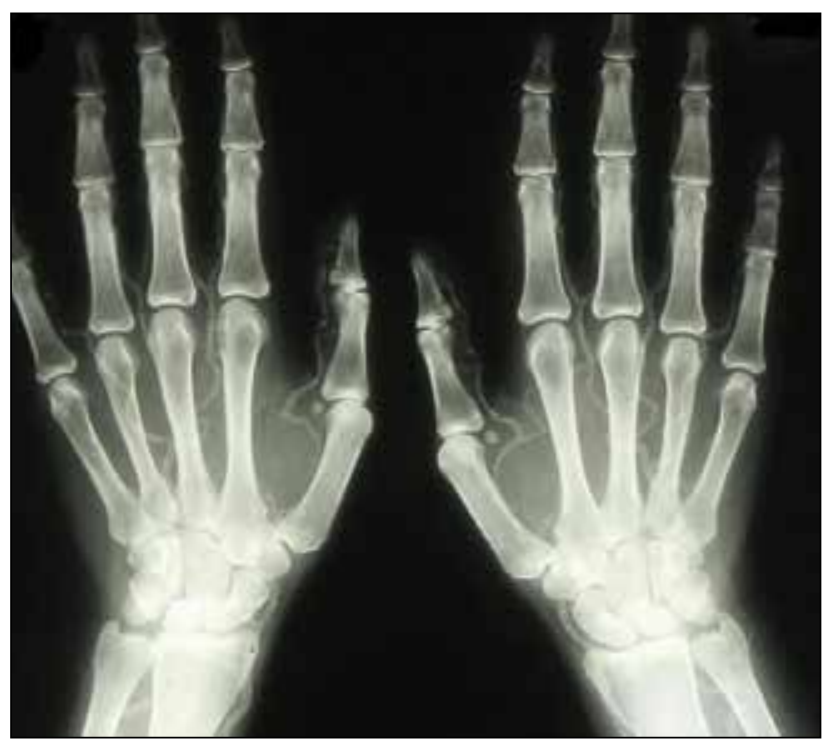

Fig. 2. Radiographs of the hands demonstrating extensive vascular calcification.

$(0.7 \%)$ and those of mixed ethnicity (1.0\%). This has also been reported by other investigators. ${ }^{[8,11]}$

The patients described in this study had several potential risk factors for calciphylaxis, ${ }^{[9,10]}$ including female gender and derangements of calcium and phosphate metabolism. Trigger factors such as the use of calcium-based phosphate binders, parenteral iron supplementation and warfarin may also have contributed to the development of calciphylaxis. ${ }^{[5,20]}$

An unusual feature of our study is the fact that most of the patients had been renal allograft recipients. It must be pointed out that in our resource-limited setting only patients suitable for transplantation are admitted to our renal replacement programme. Systemic corticosteroid use is a risk factor associated with calciphylaxis, ${ }^{[10]}$ and it is therefore possible that episodes of acute rejection and the pulse steroids used to treat these precipitated the condition in some of these transplanted patients.

The very high mortality rate is another striking feature of the study, as is the very short time from diagnosis to death. While the cause of death in patients 2, 3 and 7 could not be ascribed directly to

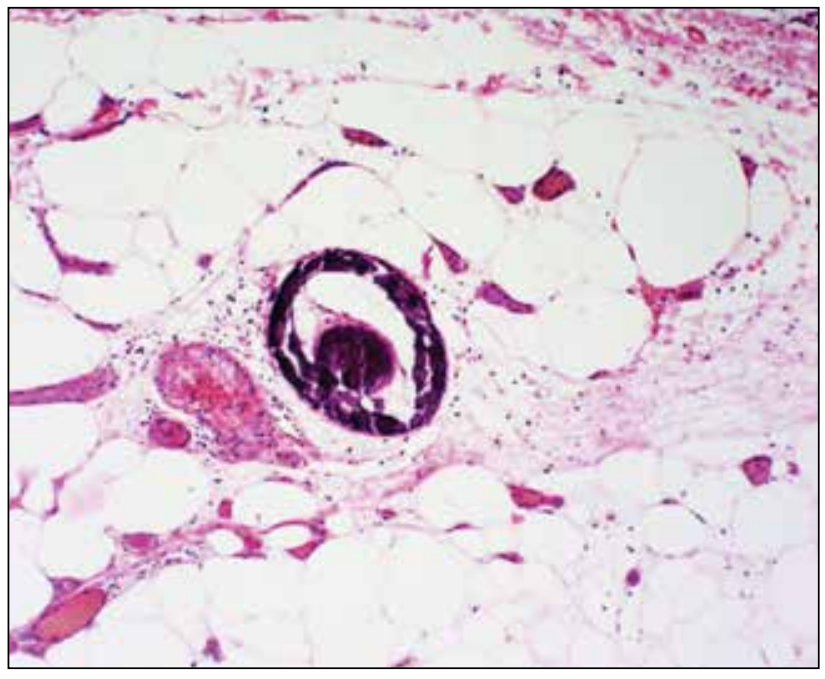

Fig. 3. Skin biopsy demonstrating circumferential microvascular calcification. Note necrosis of the adjacent subcutaneous tissue ( $H \mho E$, original magnification $\times 200$ ).

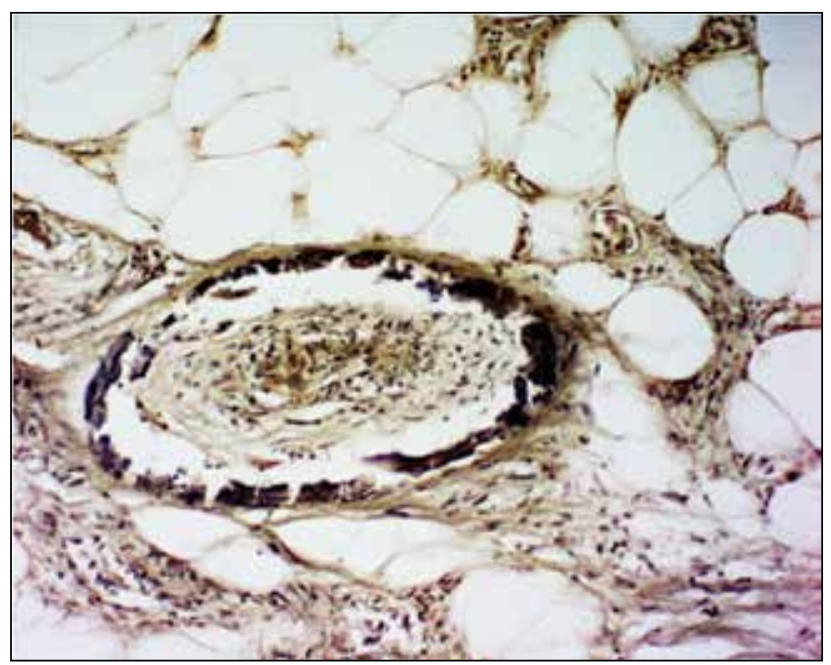

Fig. 4. Skin biopsy demonstrating circumferential microvascular calcification and an organised thrombus within the vascular lumen (Von Kossa stain, original magnification $\times 200$ ).

calciphylaxis, we nevertheless found the prognosis to be much worse than previously reported. Weenig et al.$^{[10]}$ reported a 1-year survival rate of $45.8 \%$ in their study of 64 patients, which included patients on dialysis as well as patients without ESRD. The 48 dialysis patients had a 1 -year survival rate of $29 \%$. Some authors have emphasised the distribution of the lesions, noting that patients with proximal skin lesions have a far worse prognosis than those with only distal lesions. ${ }^{[1,21]}$ Only 5 of our 19 patients fell into this category, but we nevertheless had very poor overall outcomes.

Treatment options for calciphylaxis generally remain unsatisfactory and there are no randomised trials available to guide therapy. ${ }^{[13]}$ The high cost of non-calcium-based phosphate binders, cinacalcet and sodium thiosulfate, as well as limited access to hyperbaric oxygen therapy, restricted our use of these treatment modalities. In all patients attempts were made to normalise calcium and phosphate homeostasis by stopping the use of calcium carbonate and vitamin D, switching patients to an intensified haemodialysis regimen and using bisphosphonates (one case). Good wound care, including surgical debridement, was provided 
and antibiotics were commenced at the earliest sign of local or systemic infection. A parathyroidectomy was performed on 5 patients; this seemed to contribute to wound healing in 2 cases.

\section{Conclusions}

Our experience with calciphylaxis confirms that it has a particularly poor prognosis. The high mortality rate also seems to apply to patients with distal limb lesions, which had been thought to confer a more favourable prognosis. In our cohort of patients, transplantation and the pulse steroids administered to treat episodes of acute rejection may have contributed to the development of calciphylaxis. It should always be considered in the differential diagnosis of painful skin lesions in the dialysis or transplant patient.

1. Coates T, Kirkland GS, Dymock RB, et al. Cutaneous necrosis from calcific uremic arteriolopathy. Am J Kidney Dis 1998;32(3):384-391. http://dx.doi.org/10.1053/ajkd.1998.v32.pm9740153

. Nigwekar SU, Wolf M, Sterns RH, Hix JK. Calciphylaxis from nonuremic causes: A systematic review. Clin J Am Soc Nephrol 2008;3(4):1139-1143. http://dx.doi.org/10.2215/cjn.00530108

3. Angelis M, Wong LL, Myers SA, Wong LM. Calciphylaxis in patients on hemodialysis: A prevalence study. Surgery 1997;122(6):1083-1090. http://dx.doi.org/10.1016/s0039-6060(97)90212-9

4. Selye H. Calciphylaxis. Chicago, Ill.: University of Chicago Press, 1962.

5. Weenig RH. Pathogenesis of calciphylaxis: Hans Selye to nuclear factor kappa-B. J Am Acad Dermatol 2008;58(3):458-471. http://dx.doi.org/10.1016/j.jaad.2007.12.006

6. Hayashi M. Calciphylaxis: Diagnosis and clinical features. Clin Exp Nephrol 2013;17(4):498-503. http://dx.doi.org/10.1007/s10157-013-0782-z

7. Jeong HS, Dominguez AR. Calciphylaxis: Controversies in pathogenesis, diagnosis and treatment. Am J Med Sci 2016;351(2):217-227. http://dx.doi.org/10.1016/j.amjms.2015.11.015

8. Nigwekar SU, Kroshinsky D, Nazarian RM, et al. Calciphylaxis: Risk factors, diagnosis, and treatment. Am J Kidney Dis 2015;66(1):133-146. http://dx.doi.org/10.1053/j.ajkd.2015.01.034
Am
9. Rogers NM, Coates PTH. Calcific uraemic arteriolopathy: An update. Curr Opin Nephrol Hypertens 2008;17(6):629-634. http://dx.doi.org/10.1097/mnh.0b013e32830f4566

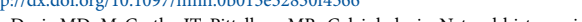
Weenig RH, Sewell LD, Davis MD, McCarthy JT, Pittelkow MR. Calciphylaxis. Natural history, risk factor analysis, and outcome. J Am Acad Dermatol 2007,56(4).569-579. http://dx.doi.org/10.1016/7. jaad.2006.08.065

1. Bleyer AJ, Choi M, Igwemezie B, de la Torre E, White WL. A case control study of proximal calciphylaxis. Am J Kidney Dis 1998;32(3):376-383. http://dx.doi.org/10.1053/ajkd.1998.v32.pm9740152

12. Galloway PA, El-Damanawi R, Bardsley V, et al. Vitamin K antagonists predispose to calciphylaxis in patient with end-stage renal disease. Nephron 2015;129(3):197-201. http://dx.doi.org/10.1159/000371449

13. Vedvyas C, Winterfield LS, Vleugels RA. Calciphylaxis: A systematic review of existing and emergin therapies. J Am Acad Dermatol 2012;67(6):e253-e260. http://dx.doi.org/10.1016/j.jaad.2011.06.009

14. Girotto JA, Harmon JW, Ratner LE, et al. Parathyroidectomy promotes wound healing and prolong survival in patients with calciphylaxis from secondary hyperparathyroidism. Surgery 2001;130(4):645651. http://dx.doi.org/10.1067/msy.2001.117101

15. Shiraishi N, Kitamura K, Miyoshi T, et al. Successful treatment of a patient with severe calcific uremic arteriolopathy (calciphylaxis) by etidronate disodium. Am J Kidney Dis 2006;48(1):151-154. http:// dx.doi.org/10.1053/..ajkd.2006.04.062

16. Velasco N, MacGregor MS, Innes A, MacKay IG. Successful treatment of calciphylaxis with cinacalcet - an alternative to parathyroidectomy? Nephrol Dial Transplant 2006;21(7):1999-2004. http:// cet - an alternative to parathys

17. An J, Devaney B, Ooi KY, et al. Hyperbaric oxygen in the treatment of calciphylaxis: A case series and literature review. Nephrology 2015;20(7):444-450. http://dx.doi.org/10.1111/nep.12433

8. Meissner M, Kaufmann R, Gille J. Sodium thiosulphate: A new way of treatment for calciphylaxis? Dermatology 2007;214(4):278-282. http://dx.doi.org/10.1159/000100877

9. Russo D, Capuano A, Cozzolino M, et al. Multimodal treatment of calcific uraemic arteriolopathy (calciphylaxis): A case series. Clin Kidney J 2016;9(1):108-112. http://dx.doi.org/10.1093/ckj/sfv120

20. Sewell LD, Weenig RH, Davis MD, McEvoy MT, Pittelkow MR. Low-dose tissue plasminogen activator for calciphylaxis. Arch Dermatol 2004;140(9):1045-1048. http://dx.doi.org/10.1001/archderm.140.9.1045

21. Duffy A, Schurr M, Warner T, Chen H. Long-term outcomes in patients with calciphylaxis from hyperparathyroidism. Ann Surg Oncol 2006;13(1):96-102. http://dx.doi.org/10.1245/aso.2006.03.042

Accepted 12 October 2016. 